\title{
Characteristics of Public Space Behavior and Demand of Rural Residents in Foshan
}

\author{
Chenyu Xie ${ }^{1, *}$ \\ ${ }^{1}$ Department of Landscape Architecture, Foshan University, Foshan, Guangdong, 528000, China
}

\begin{abstract}
With the implementation of a rural revitalization plan, rural public space has received increased attention. This article summarizes the characteristics and needs of public space behavior of residents in Dushugang village, Foshan, based on data gathered and analyzed through questionnaire survey and organized interview. The inhabitants of Dushugang village's public space behavior are characterized by six characteristics: universality and spontaneity, high frequency and comprehension, proximity, relative regularity, strong preference and universality of activity choice, and high motivation to participate in the transformation. The people of Dushugang village's public space demand can be summarized as follows: revitalizing traditional public spaces and fully explore their use; reasonable allocation and management of facilities in public spaces to optimize the landscape environment; preserving the heritage of the village culture and the healthy development of public space. This article aims to investigate the characteristics and needs of rural residents in public spaces, identify some issues in the rural construction process, and offer a new perspective on how to optimize rural transformation.
\end{abstract}

\section{Introduction}

With the continuous promotion of comprehensive deepening reform, China's urban and rural construction has made significant development. The report of the 19th National Congress points out that the principle contradiction at the current stage of our society is between the people's growing need for a better life and unbalanced and insufficient development. The people's life is constantly improving, and their demand for spiritual and cultural life is also increasing, while public space, as an essential carrier for people to pursue spiritual and cultural enjoyment in their daily lives, is receiving more and more attention. At this stage, the construction of public space in China's cities has been practical, and the countryside has seen some development under the influence of urbanization. Still, it has also brought about specific negative effects. As a result, a series of national policies have been introduced to solve the problems that have arisen in the process of rural development and to improve the quality of the living environment of rural residents.

In October 2017, China proposed the rural revitalization strategy for the first time, pointing out the need to build a new socialist countryside with flourishing industries, pleasant ecology, civilized country, effective governance, and affluent living. In 2019, the State Council proposed in the government work report that "we should do a good job of coordinating the development of urban and rural areas and accelerate the formation of a positive interaction pattern." In January 2020, the Central Committee of the Communist Party of China (CPC) and the State Council proposed that the critical work in the "three rural issues" should be carried out to ensure that the goal of overall prosperity can be achieved on schedule and that the shortcomings in infrastructure and public services should be expedited in the construction of rural areas. The introduction of many policies signifies that the development of rural construction has entered a new level.

This article focuses on rural public space. Through the research and interviews, it analyses and researches the behavioral characteristics and public space needs of rural residents, finds some problems in rural construction, and provides a new perspective for the optimization and transformation of rural public space. With the continuous promotion of urbanization, the traditional appearance of Foshan's countryside has been impacted, and it is urgent to solve the problem of how to balance the tradition and modernity of public space. Most of the current research on rural public space in Foshan is concentrated in the fields of sociology and folklore, mainly on ancestral halls, and there is relatively little comprehensive research on public space. The research in this article will play an important supplementary role in the comprehensive study of rural public space in Foshan and provide new analytical ideas for future rural planning.

\section{Rural Public Space}

Rural public space is an important place for social interaction and recreational activities for rural residents in addition to their productive work. Public space is both

*Corresponding author: chenyu.xie@outlook.com 
"publicity" and " spatiality " in nature. "Publicity" is reflected in the fact that it provides villagers with a place for public life and social interaction and is a non-material cultural carrier. The "spatiality" refers to the continuous integration of the natural environment, practical functions, folk customs, and other factors of the public space, which can develop into the spiritual support of the villagers. ${ }^{[1]}$

\subsection{Review of Research in Foreign Countries}

The study of public space first began with the concept of "public sphere" proposed by the German sociologist Habermas, who emphasized the public sphere's function of communication and dissemination. ${ }^{[2]}$ In the $1950 \mathrm{~s}$, 'public space' began to appear as a specific term in American political and sociological writings. In the early 1960s, the definition of "public space" appeared in a number of works such as The Life and Death of Great American Cities, and the concept of "public space" was formally introduced into the field of urban planning. ${ }^{[3]}$ The concept of 'public space' was officially introduced into the field of urban planning. ${ }^{[3]}$ It was only after the 1970s that the concept of 'public space' was embraced by academics and extensively researched and discussed. ${ }^{[4]}$ In 2000, the English government focused on the public living space in Our Countryside: The Future, focusing on the public space needs of residents and increasing attention to rural public space. ${ }^{[5]}$ In order to promote the healthy development of the countryside, Japan has introduced a series of policies advocating the shaping of rural public spaces with local characteristics based on the premise of loving one's hometown ${ }^{[6]}$. Germany has applied the concepts of sharing, greenery, and coordination to the landscape design of rural public spaces, summarizing the practicability of this concept through practical application.

\subsection{Review of Research in China}

Research on 'public space' in China first appeared in the 1980s in urban planning and architecture. Since the 21st century, academic research on public space has gradually extended to the countryside. A system of research on rural public space has been established and is becoming increasingly sophisticated. The discourse on rural public space in the fields of sociology and political science has mainly focused on the reconstruction of rural social order and the integration of social capital, with the views of Cao Hailin and $\mathrm{Li}$ Xiaoyun being more representative, advocating the reconstruction of rural public space through organizational, cultural and planning construction in order to promote the development of rural society. ${ }^{[7]}$ The field of urban planning and architecture mainly focuses on the function of rural space, tourism development, and landscape design. Liu Yongkang's " Research on Optimized Design of Public Space in Traditional Villages from the perspective of Public Participation - Taking Liugou Village in Beijing as an example " explores the application of public participation theory in the process of transforming rural public space, intending to transform and design public spaces that meet the needs of villagers. ${ }^{[8]}$ Bao Yafang et al.'s ' "Residents'
Perception on Factors Impacting Cultural Vitality of Public Spaces in Traditional Village of Lanxi City, Zhejiang Province " based on residents' perceptions' explores and analyses the factors influencing the cultural vitality of public space in traditional villages through exploratory factor analysis and regression analysis, to provide suggestions for improving the cultural vitality of public space ${ }^{[9]}$.

\section{Material and Method}

\subsection{Research Object}

Dushugang Village is located in the northeast of Lubao Town, Sanshui District, Foshan City, Guangdong Province. The village is surrounded by river gorges, with a total area of 19.34 square kilometers, more than $6,800 \mathrm{mu}$ of arable land, and about 5,000 mu of fish ponds. Dushugang Village has a long history, and there are many religious sites such as ancestral halls and ancient temples scattered around the village. The village consists of four natural villages, each with a communal shrine in the center of its settlement, which is an important place for conducting clan affairs and worshipping ancestors in the village. Lubao Town is a town with tourism characteristics in Guangdong Province and has a well-developed external road transport network. It is less than 50 kilometers and 45 minutes from Lubao Town to Guangzhou Baiyun International Airport and Guangzhou City and is an important link between Gaoming District and Guangzhou City. Dushugang Village is the largest village in Luzbao Town and Sanshui District, with a resident population of over 6,500. This article focuses on the characteristics of the daily spatial behavior and public space needs of rural residents in Foshan, and the selection of Dushugang Village as the research object has certain typicality and representativeness.

\subsection{Research Method}

This article mainly adopts literature review, questionnaire research, structured interviews, fieldwork, and analytical induction methods. The spatial behavioral characteristics and public space needs of villagers in Dushugang Village were also quantitatively analyzed with Questionnaire Star and SPSS AU. 


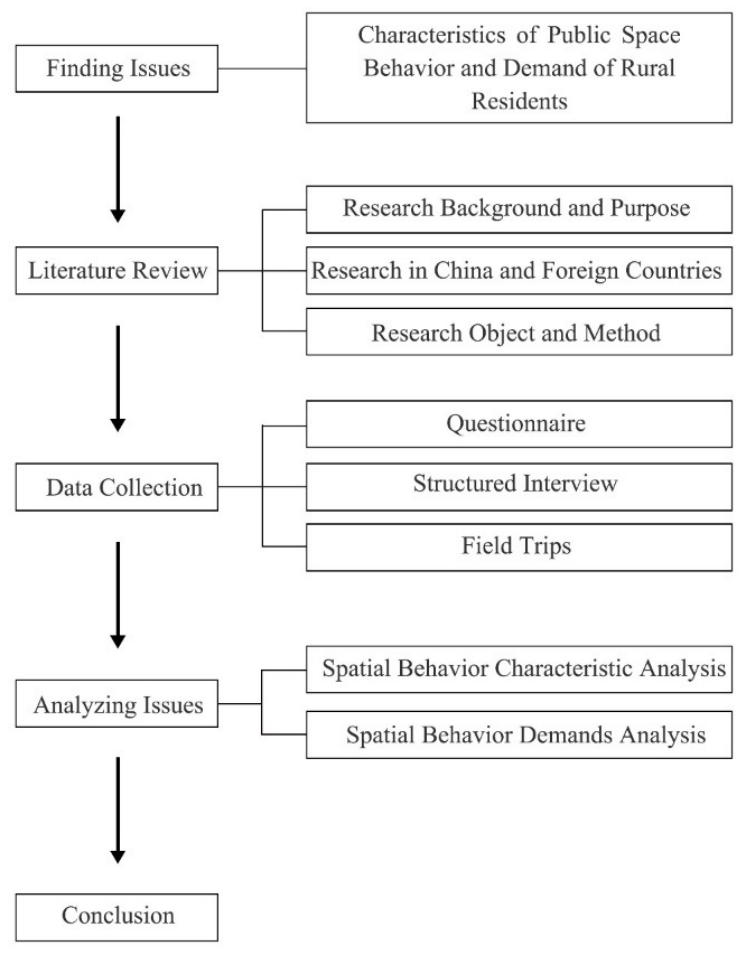

Figure 1. Technical road map

\section{Characteristics of Public Space Behavior and Demand of Residents}

\subsection{Basic Information about the Respondents}

The research on the public space in Dushugang Village consisted of two main parts: the behavioral characteristics and needs of the residents of the public space. The questionnaires and interviews were conducted online and lasted nearly a week. A total of 242 questionnaires were returned, and 40 interviews were recorded. A total of 242 people were surveyed, and the basic information of the respondents included the following four aspects: gender, age, education level, and monthly income. Age, education level, and monthly income were all divided into five classes.

Table 1. Basic information about the respondents

\begin{tabular}{|c|c|c|c|c|c|}
\hline & Quantity & Percentage & & Quantity & Percentage \\
\hline Gender & & & \multicolumn{3}{|c|}{ Education Level } \\
\hline Male & 127 & $52.5 \%$ & Primary & 44 & $18.3 \%$ \\
\hline \multirow[t]{4}{*}{ Female } & 115 & $47.5 \%$ & Junior High & 47 & $19.4 \%$ \\
\hline & & & Senior High & 76 & $31.4 \%$ \\
\hline & & & Undergraduate & 65 & $26.8 \%$ \\
\hline & & & Postgraduate & 10 & $4.1 \%$ \\
\hline Age & & & Monthly Incom & e $(\mathrm{CNY})$ & \\
\hline$<18$ & 38 & $15.7 \%$ & $<1500$ & 62 & $25.7 \%$ \\
\hline $19-28$ & 61 & $25.2 \%$ & $1500-3000$ & 31 & $12.8 \%$ \\
\hline $29-40$ & 57 & $23.6 \%$ & $3001-6000$ & 66 & $27.2 \%$ \\
\hline $41-60$ & 53 & $21.9 \%$ & $6001-8000$ & 55 & $22.7 \%$ \\
\hline$>60$ & 33 & $13.6 \%$ & $>8000$ & 28 & $11.6 \%$ \\
\hline
\end{tabular}

\subsection{Characteristics of Public Space Behavior of Residents}

Behavior is defined as the activities performed by people in response to their own needs. This article analyses the public space behavior of residents from the spatial behavior of people to analyze their behavioral changes and the relationship between the space they belong. The public spatial behavior of the residents of Dushugang Village is mainly reflected in various types of public spaces and public activities, which are primarily manifested in the following points:

(1) Universality and spontaneity. This feature is mainly reflected in the public activities carried out by the villagers. There are two main types of public activities in Dushugang village: daily public activities, such as walking in the garden and chatting at the door, and nondaily public activities, for example, the "annual festival" on the 9th day of the first month. The former is spontaneous and is a public event arranged by the people themselves, while the latter is a public activity that the whole village will participate in, and it is universal.

(2) High frequency and comprehension. The majority of residents in Dushugang Village have a high frequency of activity in public spaces, and almost all types of public spaces have traces of villagers' behavior, most residents have a high level of understanding of the distribution and environmental characteristics of public spaces in the village. There is a positive correlation between activity frequency and knowledge.

(3) Proximity. The public space behavior of the residents of Dushugang Village is characterized by proximity, which is reflected in the residents' choice of public activity locations. In their daily lives, most residents choose public spaces within one kilometer of where they live for their activities. Mountains and reservoirs, which are far away from the settlement, are less frequently selected by residents.

(4) Relative regularity. Most of the residents in Dushugang Village choose to do public activities in the afternoon and evening, and the duration of these activities lasts about half an hour. The choice of time and duration is relatively fixed.

(5) Preference and universality of activity selection. The traditional cultural activities of Dushugang village have an important place in the residents' memory, who show a clear preference for tradition in the choice of cultural activities. Moreover, the activities chosen, such as traditional religious celebrations and cultural and theatrical performances, are mostly universally applicable.

(6) High motivation to participate in the transformation. The current public space in Dushugang Village is becoming increasingly challenging to meet the residents' aspirations and pursue a better life. The residents are eager for the government and relevant organizations to transform the public space, and they are also enthusiastic about participating in it.

\subsection{Demands of Residents}

Maslow's hierarchy of needs theory suggests that people will continue to pursue higher-level needs after the lower- 
level needs are satisfied. With the continuous development of the economy, rural residents pay more and more attention to their own needs for public life $\mathrm{e}^{[10]}$. The research and interviews were conducted to analyze the public space demands of residents from two aspects: "residents' demand for public space landscape environment" and "analysis of the types of space and spatial characteristics that residents need to use." The public space demands of the residents of Dushugang Village are mainly reflected in the configuration and management of the landscape environment and facilities of various types of public space, which are mainly manifested in the following points:

(1) Revitalizing traditional public spaces and fully explore their use. There are many traditional public spaces in Dushugang Village, which have been gradually lost under the impact of urbanization and are unable to adapt to the changes in residents' attitudes and lifestyles. Residents hope that these spaces can be repaired and renovated to highlight their characteristics and that services and facilities can be rationally configured to expand their functions and change their fate of being forgotten and unused.

(2) Reasonable allocation and management of facilities in public spaces to optimize the landscape environment. Residents have the following requirements for the service facilities and landscape environment in public spaces: humane, convenient for residents to interact and communicate with their neighbors and friends; comfortable, so that people can relax physically and mentally; good sanitary conditions, high quality of safety, conducive to the activities of the elderly and children; beautiful landscape environment.

(3) Preserving the heritage of the village culture and the healthy development of public space. The residents of Dushugang Village aspire to modern public life but also hope that the unique village culture can be passed on. In the process of transforming the public space of the village, it is necessary to adapt to local conditions, improve the cultural vitality of the public space, and grasp the balance between modern construction and historical and cultural inheritance.

\section{Conclusions}

Public space is a concept that combines both 'publicity' and 'spatiality', and is influenced by the economic and cultural factors of the area in which it is located. Foshan's rural public space is currently experiencing a conflict between modern thinking and the traditional culture of the village, and the villagers have been in a passive position amid this remarkable contrast. The supply and demand of public space in Dushugang Village are in a relatively unbalanced state. The village is still dominated by the construction of large-scale housing estates, with the development of public space lagging and unable to meet the increasingly diverse needs of residents. Traditional public space areas are still difficult to survive and develop due to the restrictions of property rights. It will take a long process to reshape rural public space and enhance rural cultural vitality.

Due to the limited time and effort, this article mainly selected one village for the study, and the analysis and conclusion made in this article have certain limitations, which need to be combined with more relevant studies to create a more scientific analysis. In the process of data collection, due to the impact of the epidemic, questionnaires and structured interviews were conducted online, and the comprehensiveness of data information needs to be enhanced. The perspective and methods of analyzing data need to be explored in depth.

Each village is an independent existence, and the optimization and transformation of its internal public space cannot be generalized. We need to conduct an indepth field study and research under the guidance of relevant theories, gain an in-depth understanding of the wishes of village residents, pay attention to the production and life of the village, and adapt it to local conditions to better to promote the revitalization and development of the village.

\section{References}

1. Shi X.J. (2019) Study on the concept and Transformation of Rural Public Space. Economic Research Guide, 20: 19-23.

2. Habermas J. (1989) The structural transformation of the public sphere: an inquiry into a category of bourgeois society. The MIT Press, Cambridge.

3. Jacobs J. (1992) The Death and Life of Great American Cities. Vintage Books, New York

4. Heidelbaugh N.D., Smith M.C., Rambaut P.C. et al. (1971) Potential public health applications of space food safety standards. Journal of the American Veterinary Medical Association, 159(11).

5. Wood J.S. (1991) "Build, Therefore, Your Own World": The New England Village as Settlement Ideal. Annals of the Association of American Geographers, 81(1): 32-50.

6. Shoji S. (2009) The Village Community in Japan. Journal of Rural Studies, 15(2): 35-45.

7. Cao H.L. (2006) Village reconstruction and the reconstruction of harmonious rural communities. Social Science Research, 2: 119-123.

8. Liu Y.K. (2019) Research on Optimized Design of Public Space in Traditional Villages from the perspective of Public Participation - Taking Liugou Village in Beijing as an example. Beijing University of civil engineering and architecture.

9. Bao Y.F., Sun Z., Song M.K. et al. (2019) Residents' Perception on Factors Impacting Cultural Vitality of Public Spaces in Traditional Village of Lanxi City, Zhejiang Province, 38(5):175-180.

10. Gayle J.B., Searle F.R. (1980) Maslow, motivation and the manager. Management world, 19(9): 19-20 